\title{
Increased salience of gains versus decreased associative learning differentiate bipolar disorder from schizophrenia during incentive decision making
}

\author{
P. Brambilla ${ }^{1,2}$, C. Perlini ${ }^{3}$, M. Bellani ${ }^{3}$, L. Tomelleri ${ }^{3}$, A. Ferro ${ }^{3}$, S. Cerruti ${ }^{3}$, V. Marinelli ${ }^{3}$, \\ G. Rambaldelli ${ }^{3}$, T. Christodoulou ${ }^{4}$, J. Jogia ${ }^{4}$, D. Dima ${ }^{4}$, M. Tansella ${ }^{3}$, M. Balestrieri ${ }^{1}$ and S. Frangou ${ }^{4 *}$ \\ ${ }^{1}$ DISM, Inter-University Centre for Behavioural Neurosciences (ICBN), University of Udine, Italy \\ ${ }^{2}$ IRCCS 'E. Medea' Scientific Institute, Udine, Italy \\ ${ }^{3}$ Section of Psychiatry and Clinical Psychology, DMSP, Inter-University Centre for Behavioural Neurosciences (ICBN), University of Verona, \\ Italy \\ ${ }^{4}$ Section of Neurobiology of Psychosis, Institute of Psychiatry, King's College London, UK
}

Background. Abnormalities in incentive decision making, typically assessed using the Iowa Gambling Task (IGT), have been reported in both schizophrenia (SZ) and bipolar disorder (BD). We applied the Expectancy-Valence (E-V) model to determine whether motivational, cognitive and response selection component processes of IGT performance are differentially affected in SZ and BD.

Method. Performance on the IGT was assessed in 280 individuals comprising 70 remitted patients with SZ, 70 remitted patients with BD and 140 age-, sex- and IQ-matched healthy individuals. Based on the E-V model, we extracted three parameters, 'attention to gains or loses', 'expectancy learning' and 'response consistency', that respectively reflect motivational, cognitive and response selection influences on IGT performance.

Results. Both patient groups underperformed in the IGT compared to healthy individuals. However, the source of these deficits was diagnosis specific. Associative learning underlying the representation of expectancies was disrupted in SZ whereas BD was associated with increased incentive salience of gains. These findings were not attributable to non-specific effects of sex, IQ, psychopathology or medication.

Conclusions. Our results point to dissociable processes underlying abnormal incentive decision making in BD and SZ that could potentially be mapped to different neural circuits.

Received 1 January 2012; Revised 30 April 2012; Accepted 17 May 2012

Key words: Bipolar disorder, decision making, expectancy-valence, gambling, motivation, reward, salience, schizophrenia.

\section{Introduction}

Incentive decision making is a pervasive and fundamental mental operation. Incentive decision making occurs whenever a choice needs to be made between competing alternatives based on the value ascribed to this choice. Current theoretical models (Busemeyer \& Johnson, 2004; Berridge \& Kringelbach, 2008) propose that incentive decision making encompasses the following processes: (a) forming associations between stimuli and their outcomes, $(b)$ assigning them a value, $(c)$ extracting general principles from

\footnotetext{
* Address for correspondence: Dr S. Frangou, Section of Neurobiology of Psychosis, Department of Psychosis Studies, Institute of Psychiatry, King's College London, De Crespigny Park, London SE5 8AF, UK.

(Email: sophia.frangou@kcl.ac.uk)
}

contingencies to predict the outcome, $(d)$ implementing perceptual and response biases based on the assigned value, and (e) evaluating outcome and adjusting performance.

The Iowa Gambling Task (IGT; Bechara et al. 1994), a simulated gambling task, is a prototypical tool for investigating the processes underpinning incentive decision making. The IGT requires participants to select cards arranged in four decks. Each card has a monetary value that is revealed only after it has been selected, and can either be a gain or a loss. The participants' aim is to optimize their net gains across trials. Two of the decks have high rewards (gains) but also higher punishments (losses), resulting in monetary loss over time and they are therefore disadvantageous. The other two decks have lower rewards but also lower punishments, making them advantageous 
in the long term. Participants are not told about the distribution of gains and losses associated with each deck but must deduce this from experience during the task. Over several trials, healthy individuals learn to favour the advantageous cards.

It has long been recognized that poor performance in the IGT may arise from markedly different deficits in the processes that underpin incentive decision making. Busemeyer \& Stout (2002) proposed a mathematical model based on the concepts of expectancy (anticipated outcome based on previous experience) and valence (value ascribed to the outcome) to analyse trial-by-trial behaviour during the IGT. This model (detailed in the online supplementary material) yields three parameters that map on the basic component processes of incentive decision making: (a) attention to gains or losses (motivational parameter), representing the relative influence of wins and losses on the pattern of responding; $(b)$ expectancy learning (learning parameter), a measure of the ability to use associative learning over several trials to anticipate the outcome of future trials; and (c) response consistency, a measure of consistency of the pattern of responding. Based on this model, greater attention to gains would increase the tendency to choose from high gain disadvantageous decks. Individuals with poor expectancy learning would be more influenced by the most recent outcome of a trial and less by associative memories of more distant outcomes. Inconsistent or erratic choices suggest difficulties in applying learned expectancies about outcome during card selection.

The focus of the present study was to examine whether these component processes of decision making are differentially affected in schizophrenia (SZ) and bipolar disorder (BD).

There is renewed interest in incentive- or rewardbased processing in the major psychoses that reflects, in part, recent insights into the effect of dopamine on the cognitive and motivational aspects of incentive decision making (Bellani et al. 2009; Ziauddeen \& Murray, 2010). There is a great deal of evidence that dopaminergic input may be segregated into two complementary and interactive networks; striataldorsal prefrontal cortical circuits are implicated in the associative learning that underlies representation of expectancies whereas ventral prefrontal networks are involved in representing the motivational value of reward (Schultz 1998, 2002; Ursu \& Carter, 2005; Frank \& Claus, 2006). In SZ there is evidence of disruption in associative learning (Barch, 2005) whereas both disorders may have abnormalities in the representation of motivational value (Gold et al. 2008; Chandler et al. 2009; Bermpohl et al. 2010). Examination of the cognitive and motivational processes during the IGT in SZ and $\mathrm{BD}$ would therefore contribute to the ongoing debate about the shared and unique features of the major psychoses (Lawrie et al. 2010).

Our study builds upon evidence that overall performance in the IGT is abnormal in both disorders (Clark et al. 2001; Beninger et al. 2003; Ritter et al. 2004; Shurman et al. 2005; Christodoulou et al. 2006; Sevy et al. 2007; Bellani et al. 2009; Premkumar et al. 2008; Lee et al. 2009; Adida et al. 2011), although there have been negative studies (e.g. Cavallaro et al. 2003; Martino et al. 2011). Three studies to date, one in BD (Yechiam et al. 2008) and two in SZ (Sevy et al. 2007; Premkumar et al. 2008), have examined IGT performance in terms of Expectancy-Valence (E-V) parameters. Two of these studies were inconclusive (Sevy et al. 2007; Yechiam et al. 2008) whereas Premkumar et al. (2008) found that patients with SZ showed reduced expectancy learning but were comparable to controls in terms of attention to gains or losses.

Thus our tentative predictions were that, during the IGT, SZ would be associated with reduced expectancy learning, the cognitive component of the task, whereas BD would be associated with increased attention to gains or losses, the motivational component of the task. To test these hypotheses we compared 70 patients with SZ to 70 patients with BD and 140 healthy individuals. We enrolled patients who were in remission to reduce the potential confounding effects of symptoms (Clark et al. 2001; Sevy et al. 2007; Yechiam et al. 2008; Adida et al. 2011). Patients were matched to healthy individuals on age, sex and general intellectual ability (IQ) to minimize the influence of demographic and non-specific cognitive factors on task performance (Toplak et al. 2010).

\section{Method \\ Sample}

Participants were recruited at two sites at the University of Verona (UoV), Italy and at the Institute of Psychiatry (IoP), King's College London, UK, as part of an ongoing collaboration (Tomelleri et al. 2009). This enabled us to enrol sufficient numbers of remitted patients. Based on our previous work (Christodoulou et al. 2006; Premkumar et al. 2008), we estimated a sample size of 70 patients in each diagnostic group for a power of 0.80 and an $\alpha$ level of $<0.05$. At both sites, patients were recruited by clinicians' referrals if they (a) were aged 18-65 years and (b) fulfilled DSM-IV-R (APA, 1994) criteria for SZ or BD type I. Age-, sex- and IQ-matched healthy individuals without a personal history of any Axis I disorder or a family history of SZ or BD (up to second-degree relatives) were recruited from the respective local communities through advertisement. Exclusion criteria for the entire sample 
(patients and controls) included (a) head trauma resulting in loss of consciousness, $(b)$ personal history of neurological or medical disorders, $(c)$ family history of hereditary neurological disorders, and (d) fulfilling DSM-IV criteria for lifetime drug or alcohol dependence and drug or alcohol abuse in the preceding 6 months. The study was approved by the joint South London and Maudsley and IoP National Health Service (NHS) Research Ethics Committee and the Biomedical Ethics Committee of the Azienda Ospedaliera of Verona. Written informed consent was obtained from all participants.

\section{Clinical assessment}

At both sites clinical assessment of all participants was conducted by trained psychiatrists using structured diagnostic interviews. Diagnostic reliability (0.958) between sites was confirmed by bilingual psychiatrists on a random selection of 14 patients $(10 \%$ of the sample). At the $\mathrm{UoV}$, diagnoses were obtained using the Italian version of the Item Group Checklist of the Schedule for Clinical Assessment in Neuropsychiatry (IGC-SCAN) because the group had extensive experience with this instrument and had contributed to its standardization in Italy (Peruzzo et al. 2011). At the IoP, diagnostic assessments were performed using the SCID, patient and non-patient editions (SCID-I/P and SCID-I/NP; First et al. 2002a,b). At both sites, psychopathology was assessed using the 24-item Brief Psychiatric Rating Scale (BPRS; Luckoff et al. 1986) in addition to more disorder-specific instruments: the Hamilton Depression Rating Scale (HAMD; Hamilton, 1960) and the Young Mania Rating Scale (YMRS; Young et al. 1978) for patients with BD and the Positive and Negative Syndrome Scale (PANSS; Kay et al. 1987) for patients with SZ. The BPRS was chosen as the primary measure of psychopathology as it is applicable to both patient groups and to non-psychiatric populations.

Information about current type and dose of medication was collected for all patients. The comparable daily dose in chlorpromazine equivalents (CPZE) was calculated for current antipsychotic use when relevant (Bezchlibnyk-Butler \& Jeffries 2000).

\section{Cognitive assessment}

Both sites used the same cognitive battery. Evaluations were conducted by trained psychologists when patients were in remission, which was defined as scoring $\leqslant 3$ on the positive and negative BPRS items on the day of the assessment (Nuechterlein et al. 2006). In the BPRS, symptoms are rated from 1 (absent) to
7 (extremely severe). Item scores of 2 and 3 correspond to very mild and mild symptoms.

An estimate of IQ was obtained using the languageappropriate version of the Wechsler Adult Intelligence Scale-Revised (WAIS-R; Wechsler, 1981; Orsini \& Laicardi, 1997). Assessment of incentive decision making was based on the IGT and the Wisconsin Card Sorting test (WCST; Nelson, 1976) was used to examine non-incentive executive function. The computerized versions of the WCST and the IGT used here were previously validated in studies of $\mathrm{BD}$ and $\mathrm{SZ}$ (Donaldson et al. 2003; Frangou et al. 2005, 2006; Christodoulou et al. 2006; Premkumar et al. 2008; Ruberto et al. 2011). These tests were administered at both sites in a fixed order as reported here.

The performance of patients with $\mathrm{SZ}$ or $\mathrm{BD}$ on the WCST has been examined extensively (Stefanopoulou et al. 2009). The sole purpose of including the WCST here is to serve as a non-incentive decision-making task to aid in interpreting potential group differences between motivational and associative learning dimensions of decision making. The WCST version used consists of four stimulus cards [displaying different shapes (crosses, circles, triangles or stars) in different colours and different numbers] and a single deck of 36 response cards with displays similar to the stimulus cards. Participants were asked to match each response card to one of the stimulus cards on the basis of a series of categories (shape, colour or number) about which they had no a priori knowledge. After each selection, feedback was given and a different response card was displayed. The card-sorting category changed after a number of correct responses. The WCST requires participants to use outcome-feedback to form abstract notions of 'categories' for matching stimuli. In this respect the processes of decision making during the WCST closely approximate those involved in the IGT except that the incentive value of the WCST stimuli is very low. At the initial stages of both tasks, participants chose cards randomly as they are not given any information about stimulus-outcome associations. Following feedback, participants gradually learn the stimulus-outcome associations and adapt their responses accordingly. We therefore expected that categories achieved in the WCST would correlate with expectancy learning in the IGT.

During the IGT participants were instructed to choose a card from four decks (A, B, C, D). Each deck had different reward/punishment profiles about which participants had no a priori knowledge. At the beginning of the task, participants received a loan of pretend money and were asked to optimize their gains through their card choices. Each deck had 60 cards and participants were given 100 trials. Decks A and B were 'disadvantageous' as deck A had frequent, 
small-magnitude punishments and deck B had infrequent, but higher, punishments. By contrast, decks $C$ and D were 'advantageous' as deck C had frequent, small rewards, and deck D had infrequent, higher rewards. Dividing card selections into five blocks of 20 allowed us to monitor progress over the course of the task. Performance was evaluated using (a) the net score, calculated for each block by subtracting the total number of disadvantageous decks chosen from the total number of advantageous decks $[(C+D)-$ $(\mathrm{A}+\mathrm{B})],(b)$ the global net score, which is the sum of the net scores per block, and (c) parameters based on the E-V model. Trial number, card selected in each trial and the associated monetary loss or gain were imported into a text file and analysed in Matlab 6.1 (MathWorks, USA), as described previously (Premkumar et al. 2008) and detailed in the online supplementary material, to calculate the model's three components, as follows. (a) Attention to gains or losses (motivational parameter): this parameter ranges from 0 to 1 . Smaller values reflect attention to losses and higher values denote attention to gains, which may lead to a preference for the high-risk disadvantageous decks. (b) Expectancy learning (learning parameter): the expectancy of each deck is adjusted based on the outcome of previous experience of choosing this deck and on the payoff of the most recent choice. This parameter ranges from 0 to 1 . Small values indicate small adjustments and larger values reflect greater adjustments influenced by the outcome of the most recent choice rather than past experience. (c) Response consistency (response-sensitivity parameter): this parameter ranges from -5 (random choices) to +5 (consistent choices that are highly dependent on expectancy).

\section{Statistical analysis}

We used analyses of variance (ANOVAs) and $\chi^{2}$ tests to examine group differences in age, sex, IQ and BPRS total score. Performance in the IGT was first examined using a block-by-group (SZ patients, BD patients, healthy individuals) mixed factorial analysis of covariance (ANCOVA) of the net score. Additionally, separate ANCOVAs were performed to test for group differences in each of the $\mathrm{E}-\mathrm{V}$ parameters and in the WCST outcome variables. In all analyses, BPRS and IQ were retained as covariates to remove any variance attributable to non-specific effects of symptoms or IQ. For the IGT analyses, sex was included as an additional factor based on previous reports of sex differences on this task (Reavis \& Overman, 2001). Correlations between performance variables from the IGT and WCST were examined in addition to correlations between performance variables and antipsychotic medication dose at the time of testing, age of onset and duration of illness. The results of these analyses were considered significant at $p<0.005$ following Bonferroni correction for multiple testing. We also report effect sizes of selected group differences based on Cohen's $d$ (Cohen, 1998).

The effect of site was investigated in two ways. First, within each diagnostic group we compared the UoV to the IoP sample on each performance variable. All comparisons were non-significant $(p<0.7)$ and of negligible effect size $(d<0.06)$. Second, we repeated all analyses using site as an additional independent factor but no main or interactive effects of site were detected. For simplicity we report the results without including site as a factor as it had no effect on the findings. In addition to the primary analyses, we examined the influence of differences in rates of psychosis between the two diagnostic groups and in the prevalence of smoking

\section{Results \\ Sample}

Details of the demographic and clinical characteristics of the sample are presented in Table 1. Twenty-nine patients with SZ, 34 patients with BD and 61 healthy individuals were recruited at the $\mathrm{UoV}$ and the remainder at the IoP. There were no group differences in age $\left(F_{2,237}=2.10, p=0.13\right)$, sex distribution $\left(\chi^{2}=2.39\right.$, $p=0.30)$ or IQ $\left(F_{2,237}=1.17, p=0.18\right)$. The mean total scores (S.D.) for the HAMD and YMRS of the patients with BD were $1.54(1.73)$ and $0.81(0.87)$ respectively. The PANSS mean total score (s.D.) of patients with SZ was 42 (5.04). The scores on these scales were highly correlated with the total BPRS score $(r>0.7)$, which was the primary measure of psychopathology. Despite the low levels of psychopathology, there was a significant group difference in BPRS total score $\left(F_{2,237}=51.42, p<0.0001\right)$; patients with BD were less symptomatic than patients with SZ $(p=0.04)$ but both patient groups had higher scores than healthy individuals $(p<0.0001)$. The two patient groups did not differ in age of onset $(p=0.38)$ or duration of illness $(p=0.76)$. All patients were medicated with a variety of psychotropics. The majority received secondgeneration antipsychotics (SGAs; olanzapine $=51$, risperidone $=29$, quetiapine $=23$ ) ; those on firstgeneration antipsychotics (FGAs) received injectable formulations. The two patient groups did not differ in mean antipsychotic dose $(p=0.7)$ [mean CPZE (s.D.): patients with $\mathrm{SZ}=497.9$ (384.4); patients with $\mathrm{BD}=$ 495.4 (299.1)]. Patients prescribed antidepressants were on a variety of selective serotonin reuptake inhibitors (SSRIs; citalopram $=7, \quad$ sertraline $=4$, 
Table 1. Demographic, clinical and cognitive characteristics of the sample

\begin{tabular}{llcc}
\hline & $\begin{array}{l}\text { Healthy controls } \\
(n=140)\end{array}$ & $\begin{array}{c}\text { Patients with SZ } \\
(n=70)\end{array}$ & $\begin{array}{c}\text { Patients with BD } \\
(n=70)\end{array}$ \\
\hline Demographic information & & & $44.6(11.3)$ \\
Age (years), mean (s.D.) & $43.9(11.2)$ & $44.2(10.9)$ & $37: 33$ \\
Gender (M:F) & $71: 69$ & $41: 39$ & $26.1(10.5)$ \\
Clinical characteristics & & & $17.6(10.8)$ \\
Age of onset (years), mean (s.D.) & - & $25.2(10.1)$ & $27.4(0.8)$ \\
Duration of illness (years), mean (s.D.) & - & $18.8(10.9)$ & $54(77)$ \\
BPRS (total score), mean (s.D.) & $24.4(0.6)$ & $29.1(0.3)$ & $3(4)$ \\
History of psychosis, $n$ (\%) & - & $70(100)$ & $39(56)$ \\
Medication, $n$ (\%) & & & $59(84)$ \\
FGAs & - & $64(11)$ & $5(7)$ \\
SGAs & - & $3(4)$ & $8(11)$ \\
Mood stabilizers & & & $103.95(6.1)$ \\
Antidepressants & - & $99.1(5.9)$ & $5.2(1.0)$ \\
Cognitive task performance, mean (s.D.) & - & $4.3(1.1)$ & $3.2(2.4)$ \\
WAIS-R IQ & & $5.7(5.2)$ & $15.2(30.5)$ \\
WCST categories achieved & $101.5(4.6)$ & $6.6(26.7)$ & $0.56(0.2)$ \\
WCST perseverative errors & $5.6(0.85)$ & $0.42(0.3)$ & $0.16(0.1)$ \\
IGT global net score & $1.2(1.5)$ & $0.39(0.4)$ & $0.95(2.7)$ \\
IGT attention to gains or losses & $21.1(30.1)$ & $-0.18(2.9)$ & \\
IGT expectancy learning & $0.40(0.3)$ & $0.18(0.2)$ & \\
IGT response consistency & $1.1(1.4)$ & & \\
\hline
\end{tabular}

SZ, Schizophrenia; BD, bipolar disorder; M, male; F, female; S.D., standard deviation; BPRS, Brief Psychiatric Rating Scale; FGA, first-generation antipsychotic; SGA, second-generation antipsychotic; WAIS-R, Wechsler Adult Intelligence Scale Revised; WCST, Wisconsin Card Sorting Test; IGT, Iowa Gambling Task.

${ }^{a}$ Mood stabilizers include lithium and anticonvulsants.

fluoxetine $=2$ ). Three patients with $S Z$ were prescribed sodium valproate for arousal according to their notes. The mood stabilizers prescribed in the BD group were lithium $(n=31)$, sodium valproate $(n=23)$, carbamazepine $(n=1)$ and lamotrigine $(n=4)$. None of the patients were on regular benzodiazepines or anticholinergics or had taken any in the preceding 2 weeks. There was a significant difference in the proportion of participants who reported regular cigarette smoking (healthy individuals : $7 \%$; SZ patients : $89 \%$; BD patients : $65 \%$ ).

\section{WCST}

Details of participants' performance in the WCST are shown in Table 1 . There was a significant effect of group for categories achieved $\left(F_{2,276}=8.89, p=0.004\right)$ and for perseverative errors $\left(F_{2,276}=4.17, p=0.008\right)$. The effects of BPRS score and IQ were not significant $(p>0.25)$ for either analysis. Patients with SZ achieved fewer categories and made more perseverative errors than the other groups. We repeated the analysis with smoking status (smoking versus non-smoking) as an additional factor; the results remained unchanged and no significant main effect of smoking status was detected $\left(F_{2,276}=0.86, p=0.42\right)$.

\section{IGT}

Details of participants' performance in the IGT are shown in Table 1 and Fig. 1. We found a significant effect of group $\left(F_{2,276}=3.93, p=0.02\right)$ and groupby-block interaction $\left(F_{2,276}=8.06, p<0.0001\right)$ on net score. Both patient groups underperformed compared to healthy individuals, with SZ patients performing worse than the other groups. There was a significant effect of group on attention to gain or losses $\left(F_{2,276}=10.89, p=0.001\right)$, expectancy learning $\left(F_{2,276}=\right.$ $6.11, p=0.01)$ and response consistency $\left(F_{2,276}=12.16\right.$, $p<0.0001)$. There were no main or interactive effects of BRPS score, sex or IQ in any of the analyses $(p>0.1)$. Patients with BD scored higher on the attention to gains or losses parameter compared to all other groups $(p<0.04)$ but were comparable to controls in terms of expectancy learning and response consistency. By contrast, patients with SZ were comparable to controls in terms of attention to gains or losses but underperformed in terms of expectancy learning and 

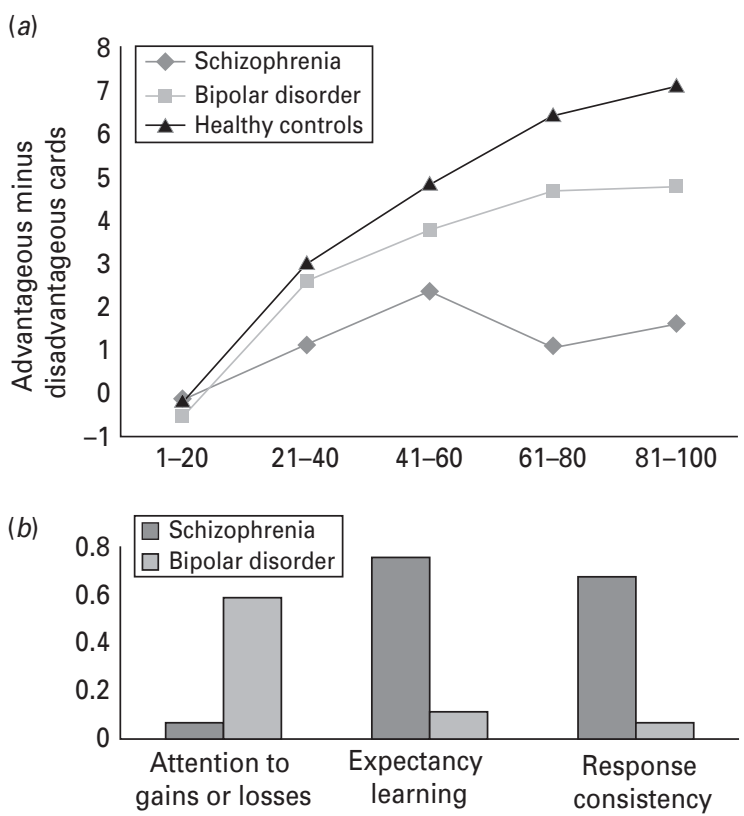

Fig. 1. (a) Performance in the Iowa Gambling Task. Net score per 20-card block; values represent group means for each block. (b) Effect size of case-control difference in Expectancy-Valence parameters for each patient group.

response consistency compared to both other groups $(p<0.001)$. We repeated the analysis adding smoking status (smoking versus non-smoking) as a factor; the results remained unchanged and no significant main effect of smoking status was detected $\left(F_{2,276}=0.30\right.$, $p=0.58)$. Within the BD group we also investigated the effect of psychosis on the E-V parameters. First, a multivariate analysis of variance (MANOVA) with the E-V parameters as dependent variables and history of psychosis as an independent factor did not reveal a significant effect of psychosis $\left(F_{1,68}=0.16, p=0.69\right)$. Second, the effect size of the difference between patients with BD with and without a history of psychosis was small for all parameters $(d \leqslant 0.26)$.

\section{Correlations between clinical and cognitive data}

For the entire sample, the global IGT net score correlated positively with categories achieved in the WCST $(r=0.35, p<0.0001)$ and inversely with the number of perseverative errors $(r=-0.24, p=0.003)$. Furthermore, categories achieved in the WCST were negatively correlated with expectancy learning $(r=-0.18, p=0.003)$. This pattern was also seen when correlations were examined within each diagnostic category separately, with absolute $r$ values of ranging from 0.24 to 0.28 and $p<0.03$. No other correlations between performance measures or with clinical measures were significant $(p>0.22)$. This also applied to correlations between the dose of current antipsychotics and the WCST and IGT outcome variables (absolute $r<0.08, p>0.24$ ).

\section{Discussion}

Our results indicate that, during the IGT, SZ and BD are associated with diagnosis-specific abnormalities in the motivational, cognitive and response selection components of the task. Patients with BD showed increased attention to gains or losses compared to both other groups. Patients with SZ had poor expectancy learning and response consistency compared to patients with BD and healthy individuals. These results were not attributable to non-specific effects of sex, IQ, psychopathology or medication.

\section{Increased incentive salience of gains in $B D$}

Our study confirms previous reports of abnormal incentive decision making in remitted BD patients (Christodoulou et al. 2006; Adida et al. 2011). Adida et al. (2011) reported that BD patients do not show aberrant responses to penalties during the IGT. Our results extend these findings to demonstrate that patients with $\mathrm{BD}$ are more motivated by gains. Our study is also comparable to that of Yechiam et al. (2008), who also applied the E-V model when comparing IGT performance between remitted BD patients $(n=14)$ and healthy controls $(n=14)$. Although their reported mean of the attention to gains or losses parameter (0.59) was identical to ours, the study was negative because it was underpowered. However, it is important to note that BD patients performed similarly to controls in terms of expectancy learning and response consistency, indicating that their ability to extract and apply expectancies representing the anticipated consequences of choosing a card from each deck was not impaired. We therefore infer that BD patients seem to 'override' these cognitive biases because of the higher 'incentive salience' placed on gains (Berridge \& Kringelbach, 2008). Our results are also in agreement with Chandler et al. (2009), who found that BD patients displayed a normal understanding of the context in which they were asked to make choices relating to gains or losses; this contextual information, however, had diminished impact on their behaviour. It is worth noting that their BD sample consisted of high-functioning (mean $I Q=122$ ), unmedicated, euthymic university students with minimal duration of illness. This implies that the decision-making strategies identified in this study are also relevant to the earlier stages of the illness and are independent of general intellectual ability. We suggest that the concept of increased incentive salience of gains provides an apt description of the behaviour of 
BD patients during the IGT as it reflects motivational attitudes that are largely separable from contextual information and planning (Berridge \& Kringelbach, 2008). Although our findings capture trait strategies in incentive decision making in $\mathrm{BD}$, mood state at the time of testing seems to have a significant impact on these strategies. The presence of depression may increase the salience of negative outcomes (Roiser et al. 2009; Adida et al. 2011) whereas mania has been associated with increasingly erratic and random choices (Minassian et al. 2004; Adida et al. 2008; Yechiam et al. 2008).

\section{Decreased expectancy learning in SZ}

SZ patients had reduced overall performance in the IGT compared to controls, which adds to the existing literature on abnormal incentive decision making in this disorder (Beninger et al. 2003; Ritter et al. 2004; Shurman et al. 2005; Sevy et al. 2007; Bellani et al. 2009; Premkumar et al. 2008; Lee et al. 2009). Confirming our previous report (Premkumar et al. 2008), we found that patients with SZ, in this independent sample, had reduced expectancy learning. This cognitive parameter of the E-V model reflects the influence of associative learning on behaviour; larger values, such as were seen in SZ patients, suggest that choices are guided by the more recent outcomes whereas past outcomes are discounted. Expectancy learning relies on monitoring the distribution of gains and losses for each deck on a trial-by-trial basis and on using this information to extract general rules (expectancies) to predict the likely outcome of card choices. As Bechara et al. (1998) have shown, the ability to form and maintain online representations of various options during incentive decision making is dependent on executive function mechanisms. The negative correlation observed between expectancy learning and categories achieved in the WCST lends further support to this notion. Our results therefore suggest that incentive decisionmaking deficits in SZ are linked to impairment in executive functions associated with rule discovery. This may also explain the lack of consistency in their choices. In contrast to BD patients, patients with SZ were comparable to controls on the attention to gains or losses parameter. We infer that SZ patients experienced similar reactions to gains and losses as controls and that underperformance in the IGT in SZ is not attributable to motivational processes. Taken together, these findings can be best understood within the framework proposed by Gold et al. (2008). According to this model, patients with SZ have deficits in incentive decision making, despite normal motivational influences, because of their reduced ability to process 'representational complexity', which refers to the process of simultaneous representation of the multiple motivational and cognitive aspects of decision making. The model further proposes that this deficit represents another facet of executive dysfunction associated with SZ.

\section{Implications for the relationship between $B D$ and $S Z$}

Decomposing the performance of patients with SZ or BD during the IGT using motivational, cognitive and response choice parameters derived from the E-V model yielded significant diagnostic differences. Associative learning underlying the representation of expectancies was disrupted in SZ, which reinforces reports of abnormalities in reward-based learning in this disorder (Gold et al. 2008), and implicates circuits linking the associative striatum with the dorsal prefrontal cortex (Corlett et al. 2007). By contrast, BD was associated with increased incentive salience of gains. This is the first study to demonstrate this in remitted BD patients. Bermpohl et al. (2010) have shown that similarly altered attention to rewards in manic BD patients was associated with enhanced activity within the ventral prefrontal cortex, a finding replicated during incentive decision making in remitted BD patients (Frangou et al. 2008; Jogia et al. 2011). Our results therefore point to dissociable abnormalities in incentive decision making in BD and SZ that could potentially be mapped to different neural circuits.

\section{Methodological considerations}

All patients were medicated at the time of testing with a variety of psychotropics. Antipsychotic medication, particularly with FGAs, is known to affect motivational and reward-based processes (Beninger et al. 2003; Juckel et al. 2006; Sevy et al. 2006; Schlagenhauf et al. 2008). At the same time, consistent with other colleagues (e.g. Cavallaro et al. 2003; Premkumar et al. 2008), we did not find significant correlations between IGT performance and antipsychotic dose. It was not possible to conduct subanalyses based on medication type alone as the size of the corresponding groups was small and therefore comparisons would not have been meaningful. There was a significant difference in the proportion of regular cigarette smokers between the diagnostic groups. However, the inclusion of smoking status as an independent factor did not alter our findings and the effect of smoking status was not statistically significant.

To summarize, we have presented data showing that trait incentive decision-making abnormalities in $\mathrm{SZ}$ and BD can be attributed to dissociable processes respectively relating to expectancy learning and attention to gains or losses. Further studies are required 
to examine the relevance of these behavioural observations to the biological correlates of these two disorders.

\section{Supplementary material}

For supplementary material accompanying this paper visit http://dx.doi.org/10.1017/S0033291712001304.

\section{Acknowledgements}

This work was partly supported by grants from the American Psychiatric Institute for Research and Education (APIRE Young Minds in Psychiatry Award), the Italian Ministry for University and Research and the Italian Ministry of Health (IRCCS 'E. Medea') to P. Brambilla.

\section{Declaration of Interest}

None.

\section{References}

Adida M, Clark L, Pomietto P, Kaladjian A, Besnier N, Azorin JM, Jeanningros R, Goodwin GM (2008). Lack of insight may predict impaired decision making in manic patients. Bipolar Disorders 10, 829-837.

Adida M, Jollant F, Clark L, Besnier N, Guillaume S, Kaladjian A, Mazzola-Pomietto P, Jeanningros R, Goodwin GM, Azorin JM, Courtet P (2011). Trait-related decision-making impairment in the three phases of bipolar disorder. Biological Psychiatry 70, 357-365.

APA (1994). Diagnostic and Statistical Manual of Mental Disorders: DSM-IV. American Psychiatric Association: Washington, DC.

Barch DM (2005). The relationships among cognition, motivation, and emotion in schizophrenia: how much and how little we know. Schizophrenia Bulletin 31, 875-881.

Bechara A, Damasio AR, Damasio H, Anderson SW (1994). Insensitivity to future consequences following damage to human prefrontal cortex. Cognition 50, 7-15.

Bechara A, Damasio H, Tranel D, Anderson WS (1998). Dissociation of working memory from decision making within the human prefrontal cortex. Journal of Neuroscience 18, 426-437.

Bellani M, Tomelleri L, Brambilla P (2009). Emotion-based decision making in schizophrenia: evidence from the Iowa Gambling Task. Epidemiologia e Psichiatria Sociale 18, 104-106.

Beninger RJ, Wasserman J, Zanibbi K, Charbonneau D, Mangels J, Beninger BV (2003). Typical and atypical antipsychotic medications differentially affect two nondeclarative memory tasks in schizophrenic patients: a double dissociation. Schizophrenia Research 61, 281-292.

Bermpohl F, Kahnt T, Dalanay U, Hägele C, Sajonz B, Wegner T, Stoy M, Adli M, Krüger S, Wrase J, Ströhle A,
Bauer M, Heinz A (2010). Altered representation of expected value in the orbitofrontal cortex in mania. Human Brain Mapping 31, 958-969.

Berridge KC, Kringelbach ML (2008). Affective neuroscience of pleasure: reward in humans and animals. Psychopharmacology (Berlin) 199, 457-480.

Bezchlibnyk-Butler KZ, Jeffries JJ (2000). Clinical Handbook of Psychotropic Drugs, 10th edn. Hogrefe \& Huber: Seattle, pp. 84-88.

Busemeyer JR, Johnson JG (2004). Computational models of decision making. In Blackwell Handbook of Judgment and Decision Making (ed. D. Koehler and N. Harvey), pp. 133-154. Blackwell Publishing Co. : New York.

Busemeyer JR, Stout JC (2002). A contribution of cognitive decision models to clinical assessment. Decomposing performance on the Bechara gambling task. Psychological Assessment 14, 253-262.

Cavallaro R, Cavedini P, Mistretta P, Bassi T, Angelone SM, Ubbiali A, Bellodi L (2003). Basal-corticofrontal circuits in schizophrenia and obsessive-compulsive disorder: a controlled, double dissociation study. Biological Psychiatry 54, 437-443.

Chandler RA, Wakeley J, Goodwin GM, Rogers RD (2009). Altered risk-aversion and risk-seeking behavior in bipolar disorder. Biological Psychiatry 66, 840-846.

Christodoulou T, Lewis M, Ploubidis GB, Frangou S (2006). The relationship of impulsivity to response inhibition and decision-making in remitted patients with bipolar disorder. European Psychiatry 21, 270-273.

Clark L, Iversen SD, Goodwin GM (2001). A neuropsychological investigation of prefrontal cortex involvement in acute mania. American Journal of Psychiatry 158, 1605-1611.

Cohen J (1998). Statistical Power Analysis for the Behavioral Sciences, 2nd edn. Lawrence Erlbaum Associates: Hillsdale, NJ.

Corlett PR, Murray GK, Honey GD, Aitken MR, Shanks DR, Robbins TW, Bullmore ET, Dickinson A, Fletcher PC (2007). Disrupted prediction-error signal in psychosis: evidence for an associative account of delusions. Brain 130, 2387-2400.

Donaldson S, Goldstein LH, Landau S, Raymont V, Frangou $S$ (2003). The Maudsley Bipolar Disorder Project: the effect of medication, family history, and duration of illness on IQ and memory in bipolar I disorder. Journal of Clinical Psychiatry 64, 86-93.

First MB, Spitzer RL, Gibbon M, Williams JBW (2002a). Structured Clinical Interview for DSM-IV-TR Axis I Disorders, Research Version, Patient Edition (SCID-I/P). Biometrics Research, New York State Psychiatric Institute: New York.

First MB, Spitzer RL, Gibbon M, Williams JBW (2002b). Structured Clinical Interview for DSM-IV-TR Axis I Disorders, Research Version, Non-Patient Edition (SCID-I/NP).

Biometrics Research, New York State Psychiatric Institute: New York.

Frangou S, Dakhil N, Landau S, Kumari V (2006). Frontotemporal function may distinguish bipolar disorder from schizophrenia. Bipolar Disorders 8, 47-55. 
Frangou S, Donaldson S, Hadjulis M, Landau S, Goldstein LH (2005). The Maudsley Bipolar Disorder Project: executive dysfunction in bipolar disorder I and its clinical correlates. Biological Psychiatry 58, 859-864.

Frangou S, Kington J, Raymont V, Shergill SS (2008). Examining ventral and dorsal prefrontal function in bipolar disorder: a functional magnetic resonance imaging study. European Psychiatry 23, 300-308.

Frank MJ, Claus ED (2006). Anatomy of a decision: striato-orbitofrontal interactions in reinforcement learning, decision making, and reversal. Psychological Review 113, 300-326.

Gold JM, Waltz JA, Prentice KJ, Morris SE, Heerey EA (2008). Reward processing in schizophrenia: a deficit in the representation of value. Schizophrenia Bulletin 34, 835-847.

Hamilton MA (1960). Rating scale for depression. Journal of Neurology, Neurosurgery and Psychiatry 23, 56-62.

Jogia J, Dima D, Kumari V, Frangou S (2011). Frontopolar cortical inefficiency may underpin reward and working memory dysfunction in bipolar disorder. World Journal of Biological Psychiatry. Published online: 3 August 2011. doi:10.3109/15622975.2011.585662.

Juckel G, Schlagenhauf F, Koslowski M, Filonov D, Wüstenberg T, Villringer A, Knutson B, Kienast T, Gallinat J, Wrase J, Heinz A (2006). Dysfunction of ventral striatal reward prediction in schizophrenic patients treated with typical, not atypical, neuroleptics. Psychopharmacology (Berlin) 187, 222-228.

Kay SR, Fiszbein A, Opler LA (1987). The positive and negative syndrome scale (PANSS) for schizophrenia. Schizophrenia Bulletin 13, 261-276.

Lawrie SM, Hall J, McIntosh AM, Owens DG, Johnstone EC (2010). The 'continuum of psychosis': scientifically unproven and clinically impractical. British Journal of Psychiatry 197, 423-425.

Lukoff D, Nuechterlien K, Ventura J (1986). Manual for the expanded Brief Psychiatric Rating Scale. Schizophrenia Bulletin 12, 594-608.

Martino DJ, Strejilevich SA, Torralva T, Manes F (2011). Decision making in euthymic bipolar I and bipolar II disorders. Psychological Medicine 41, 1319-1327.

Minassian A, Paulus MP, Perry W (2004). Increased sensitivity error during decision-making in bipolar disorder patients acute mania. Journal of Affective Disorders 82, 203-208.

Nelson HE (1976). A modified card sorting test sensitive to frontal lobe defects. Cortex 12, 313-324.

Nuechterlein KH, Miklowitz DJ, Ventura J, Gitlin MJ, Stoddard M, Lukoff D (2006). Classifying episodes in schizophrenia and bipolar disorder : criteria for relapse and remission applied to recent-onset samples. Psychiatry Research 144, 153-165.

Orsini A, Laicardi C (1997). WAIS-R. Contributo alla Taratura Italiana. Organizzazioni Speciali: Firenze.

Peruzzo D, Rambaldelli G, Bertoldo A, Bellani M, Cerini R, Silvia M, Pozzi Mucelli R, Tansella M, Brambilla P (2011). The impact of schizophrenia on frontal perfusion parameters: a DSC-MRI study. Journal of Neural

Transmission 118, 563-570.

Premkumar P, Fannon D, Kuipers E, Simmons A, Frangou S, Kumari V (2008). Emotional decisionmaking and its dissociable components in schizophrenia and schizoaffective disorder: a behavioural and MRI investigation. Neuropsychologia 46, 2002-2012.

Reavis R, Overman WH (2001). Adult sex differences on a decision-making task previously shown to depend on the orbital prefrontal cortex. Behavioral Neuroscience 115, 196-206.

Ritter LM, Meador-Woodruff JH, Dalack GW (2004). Neurocognitive measures of prefrontal cortical dysfunction in schizophrenia. Schizophrenia Research 68, 65-73.

Roiser JP, Cannon DM, Gandhi SK, Taylor Tavares J, Erickson K, Wood S, Klaver JM, Clark L, Zarate Jr. CA, Sahakian BJ, Drevets WC (2009). Hot and cold cognition in unmedicated depressed subjects with bipolar disorder. Bipolar Disorders 11, 178-189.

Ruberto G, Vassos E, Lewis CM, Tatarelli R, Girardi P, Collier D, Frangou $S$ (2011). The cognitive impact of the ANK3 risk variant for bipolar disorder : initial evidence of selectivity to signal detection during sustained attention. PLOS ONE 6, e16671.

Schlagenhauf F, Juckel G, Koslowski M, Kahnt T, Knutson B, Dembler T, Kienast T, Gallinat J, Wrase J, Heinz A (2008). Reward system activation in schizophrenic patients switched from typical neuroleptics to olanzapine. Psychopharmacology (Berlin) 196, 673-684.

Schultz W (1998). Predictive reward signal of dopamine neurons. Journal of Neurophysiology 80, 1-27.

Schultz W (2002). Getting formal with dopamine and reward. Neuron 36, 241-263.

Sevy S, Hassoun Y, Bechara A, Yechiam E, Napolitano B, Burdick K, Delman H, Malhotra A (2006). Emotion-based decision-making in healthy subjects: short-term effects of reducing dopamine levels. Psychopharmacology (Berlin) 188, 228-235.

Sevy S, Burdick KE, Visweswaraiah H, Abdelmessih S, Lukin M, Yechiam E, Bechara A (2007). Iowa Gambling Task in schizophrenia: a review and new data in patients with schizophrenia and cooccurring cannabis use disorders. Schizophrenia Research 92, 74-84.

Shurman B, Horan WP, Nuechterlein KH (2005). Schizophrenia patients demonstrate a distinctive pattern of decision-making impairment on the Iowa Gambling Task. Schizophrenia Research 72, 215-224.

Stefanopoulou E, Manoharan A, Landau S, Geddes JR, Goodwin G, Frangou S (2009). Cognitive functioning in patients with affective disorders and schizophrenia: a meta-analysis. International Reviews of Psychiatry 21, 336-356.

Tomelleri L, Jogia J, Perlini C, Bellani M, Ferro A, Rambaldelli G, Tansella M, Frangou S, Brambilla P; Neuroimaging Network of the ECNP networks initiative (2009). Brain structural changes associated 
with chronicity and antipsychotic treatment in schizophrenia. European Neuropsychopharmacology 19, 835-840.

Toplak ME, Sorge GB, Benoit A, West RF, Stanovich KE (2010). Decision-making and cognitive abilities: a review of associations between Iowa Gambling Task performance, executive functions, and intelligence. Clinical Psychology Review 30, 562-581.

Ursu S, Carter CS (2005). Outcome representations, counterfactual comparisons and the human orbitofrontal cortex: implications for neuroimaging studies of decision-making. Cognitive Brain Research 23, 51-60.
Wechsler D (1981). Wechsler Adult Intelligence ScaleRevised (WAIS-R) Manual. Psychological Corporation : New York.

Yechiam E, Hayden EP, Bodkins M, O'Donnell BF, Hetrick WP (2008). Decision making in bipolar disorder: a cognitive modelling approach. Psychiatry Research 161, 142-152.

Young R, Biggs J, Zieglar V, Meyer D (1978). A rating scale for mania: reliability, validity and sensitivity. British Journal of Psychiatry 133, 429-435.

Ziauddeen H, Murray GK (2010). The relevance of reward pathways for schizophrenia. Current Opinion in Psychiatry 23, 91-96. 